\section{Wenn nachts die Luftnot kommt ...}

\section{Das Risiko für einen Asthmaanfall ist während der Nacht am höchsten. Auch die Wahrscheinlichkeit, an einem Asthmaanfall zu sterben, hat einen Gipfel zwischen zwei und vier Uhr morgens. Warum das so ist und weshalb besonders Kinder und Jugendliche von nokturnalem Asthma betroffen sind, wollten wir von Prof. Dr. Adrian Gillissen aus Kassel wissen.}

? Herr Professor Gillissen, wie häufig ist ausschließlich nächtliches Asthma? Trifft es $z u$, dass Kinder und Jugendliche hiervon besonders betroffen sind?

Gillissen: Ausschließlich nächtliches Asthma ist eher selten, zumindest im Vergleich aller Asthmatypen. Nächtliches Asthma tritt häufig beim nicht gut eingestellten oder initial noch unbekannten und daher nicht therapierten Asthma auf.

Es stimmt, dass Kinder und Jugendliche mehr an nächtlichem Asthma leiden als Erwachsene. Dies äußert sich übrigens nicht nur in nächtlichen Dyspnoe-, sondern vor allem in frühmorgendlichen Hustenanfällen, mit denen die Patienten aufwachen. Vor allem diese nächtlichen Hustensensationen werden bei Kindern häufig als viraler Infekt fehlinterpretiert. Eine erfolgreiche Therapie verhindert diese Symptome.

\section{? Wie erklärt man sich das Phänomen des nokturnalen Asthma pathophysiologisch?}

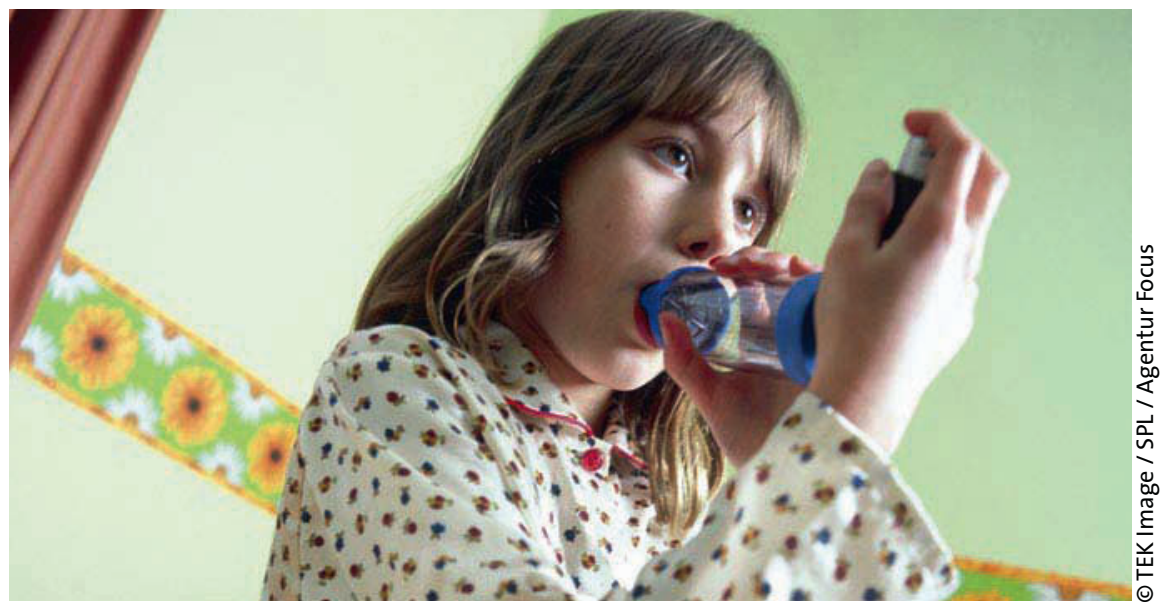

Schlecht eingestellte Asthmapatienten werden oft nachts von Atemnot aus dem Schlaf geschreckt. Chronobiologische Untersuchungen haben ergeben, dass zwischen zwei und vier Uhr morgens die Bronchialweite am geringsten ist.
Gillissen: Pathophysiologisch hängt dieses Phänomen bei entsprechend prädestinierten Patienten mit dem nachts niedrigeren Cortisol-Serumspiegel zusammen. Normalerweise führt diese physiologische Absenkung zu keinerlei Problemen.

Man nimmt aber an, dass bei einem Asthmapatienten niedrigere Cortisolspiegel eine leichte bronchiale Entzündung nicht mehr unterdrücken können, was der Patient dann in Form von frühmorgendlichem trockenem Husten - ohne Auswurf - und ggf. auch Dyspnoe oder Enge in der Brust verspürt.

? Wie wird die Diagnose gesichert? Orientiert man sich in der Praxis nur an den subjektiven Symptomen der Patienten?

Gillissen: Die wichtigste diagnostische Aussage sind natürlich die Symptome, die eine weitere Diagnostik nach sich ziehen müssen. Meistens ist bei ausschließlich nächtlichem Asthma oder bei nächtlichem asthmabedingtem Husten die Lungenfunktion tagsüber normal. Dies macht die Diagnostik schwierig. Ein pathologischer Befund im Methacholintest, bei dem die bronchiale Hyperreaktivität gemessen wird, oder in den von dem Patienten selbst durchführbaren Peak-Flow-Messungen, würde die initiale Verdachtsdiagnose eines nächtlichen Asthmas erhärten. Der nächste Schritt wäre die Einleitung einer Asthmatherapie. Ein Therapieerfolg sichert dann retrospektiv die Diagnose.

? Was ist bei dieser Asthmaform therapeutisch zu beachten?

Gillissen: Eine instabile Asthmaform, und dazu zählen natürlich auch nächtliche Husten- und Luftnotanfälle, werden am besten mit einem inhalativen kortisonhaltigen Präparat therapiert. Dieses unterdrückt die bronchiale Entzündung und führt zu einer Normalisierung der klinischen Symptomatik. So könnte man beispielsweise mit der regelmäßigen abendlichen Inhalation eines niedrig dosierten Budesonids, Beclometasons oder Fluticasons beginnen. Die Verwendung eines rasch wirksamen $\beta_{2}$-Mimetikums eignet sich nur als Bedarfsmedikament, da es die diesem Phänomen zugrunde liegende Entzündung nicht beeinflusst, sondern nur die muskuläre Konstriktion. Je häufiger ein $\beta_{2}$-Mimetikum verwendet wird oder vom Patienten verwendet werden muss, desto wichtiger ist der regelmäßige Einsatz eines inhalativen Kortikosteroids.

! Herr Professor Gillissen, vielen Dank für das Gespräch.

Das Interview führte Dr. Brigitte Moreano. 